\title{
Editor's Outlook
}

\section{Andrew J. Ball}

I am pleased to begin the final issue of the year with a very special announcement. Screen Bodies is modifying its editorial direction and the kind of work it will feature. Many of our readers will already have a sense of these changes, made evident by the new Aims and Scope section we made available online earlier this summer, and by the journal's new subtitle, The journal of Embodiment, Media Arts, and Technology. As these indicate, the foundational commitments of the journal remain unchanged; however, moving forward will we intensify our focus on new media art, technology studies, and the interface of the sciences and the humanities. We will continue to examine the cultural, aesthetic, ethical, and political dimensions of emerging technologies, but with a renewed attention to such areas as intermediality, human-machine interface, virtual and augmented reality, artificial intelligence, generative art, smart environments, immersive and interactive installations, machine learning, biotechnology, computer science, digital culture, and digital humanities. The journal will continue to prioritize matters of the body and screen media, both in terms of representation and engagement, but will emphasize research that critically reexamines those very concepts, as, for example, in the case of object-oriented feminism's nonanthropocentric approach, which asks us to rethink what we mean by bodies and embodiment.

In addition to remaining a premier platform for peer-reviewed criticism in these areas, we will be introducing a section of the journal where artists can speak for themselves. This section will include interviews and commentaries on a variety of multidisciplinary topics but will focus in particular on artist-authored works on practice. Much of this work will be commissioned, but we encourage artists to submit commentaries on their current practice.

Though the recalibration of Screen Bodies was not planned to become official until 2021, with Volume 6, both Issues 5.1 and 5.2 are exemplars of the new model. So, to get an idea of what Screen Bodies will look like, we need only turn to the outstanding work published in the journal this year. For example, in 5.1 we featured Amy Borden's excellent article on intermediality in American silent cinema, and in this issue we are pleased to present Olivier Delers's article on transmediality in Paul Chan's art project Sade for Sade's Sake. These two issues 
also give us a glimpse into many of the topics we will prioritize in the future, such as installation art, artificial intelligence, biotechnology, gaming, digital art, posthumanism, and film. And in keeping with the established emphases of the journal, these two issues feature the foremost innovations in queer theory, gender and sexuality studies, and in critical race and ethnic studies.

These many disciplinary and topical elements are elegantly assembled in this issue's special section entitled “Queer Sinofuturisms." We are particularly excited to contribute to the discourse surrounding the remarkable and still-developing concept of Sinofuturism. I will leave it to our esteemed guest editors, Ari Heinrich, Howard Chiang, and Ta-wei Chi, to introduce this fascinating topic.

In closing, I am also pleased to announce that, earlier this year, I was honored to accept the invitation to serve as the new editor of Screen Bodies. The journal's founding editor, Brian Bergen-Aurand, has built something truly special here, and I look forward to guiding Screen Bodies into its next chapter. 